\title{
SINERGIA
}

REVISTA DO INSTITUTO DE CIÊNCIAS ECONÔMICAS, ADMINISTRATIVAS E CONTÁBEIS (ICEAC)

\section{INDICADORES DE DESEMPENHO EM SERVIÇOS E PRODUÇÃO: PROPOSTA DE MAPEAMENTO DA PRODUÇÃO CIENTÍFICA À LUZ DA BIBLIOMETRIA ${ }^{1}$}

\author{
ERROL FERNANDO ZEPKA PEREIRA JUNIOR \\ LÍVIA CASTRO D'AVILA* \\ FRANCIELLE DA SILVEIRA PEREIRA
}

\begin{abstract}
O presente trabalho tem como objetivo caracterizar a produção científica referente aos indicadores de desempenho em serviços e produção no período compreendido entre 2007 e 2017, utilizando-se a bibliometria para realizar estudos na produção, no que refere à autoria, ao conteúdo e às referências dos artigos selecionados, por meio da análise de artigos disponíveis em bases de dados online. Metodologicamente, o estudo é de natureza exploratória, porquanto adota a revisão bibliográfica dos artigos. Foram buscados os artigos nas bases de dados Science Direct, Scopus e Web of Science. Através da análise bibliométrica, retiveram-se 27 artigos com maior relevância no assunto. Entre os principais resultados encontrados na análise, foi possível constatar que os temas mais pesquisados nos artigos foram a busca por indicadores de desempenho em empresas prestadoras de serviços e empresas de processos produtivos. A maioria dos artigos analisados foram escritos por 2 ou 4 autores. $E$ as universidades mais produtivas sobre o tema foram: o Korea Institute of Science and Technology Information, da Coréia e o Instituto Politecnico di Torino, da Itália.
\end{abstract}

RESUMO

Palavras-Chave: indicadores de desempenho, serviços, produção, bibliometria.

\section{ABSTRACT}

The present work pretends characterize the scientific production referring to the performance indicators in services and production in the period between 2007 and 2017, using bibliometrics to carry out studies on the production, authorship, content and references of the articles selected through the analysis of articles available in online database. Methodologically, the study is exploratory in nature, since it adopts the bibliographic review of the articles. We searched the articles in the databases Science Direct, Scopus and Web of Science. Through the bibliometric analysis, 27 articles were retained with great relevance in the subject. Among the main results found in the analysis it was possible to verify that the most researched topics in the articles were the search for performance indicators in companies that provide services and production processes. Most articles analyzed were written by 2 or 4 authors. And the most productive universities on the subject were: Korea Institute of Science and Technology Information of Korea and Institute Politecnico di Torino of Italy.

Keywords: performance indicator, services, production, bibliometry

Recebido em: 30-11-2017 Aceito em: 25-06-2018

\section{INTRODUÇÃO}

O atual ambiente de mercado tem se apresentado cada vez mais mutável e imprevisível. A consolidação da concorrência em escala global já é algo intrínseco do mercado, dessa forma, as organizações precisam buscar novas formas de lidar com o controle de desempenho para poderem se ajustar frente às novas demandas do mercado. Até a década de 70 , o pensamento gerencial se orientava para áreas particulares, como finanças, marketing, produção e outras. Todavia, com o crescimento da competitividade, torna-se imprescindível a necessidade de uma gestão sistêmica da organização (FISCHMANN e ZILBER, 1999). Um dos elementos mais básicos da gestão é o controle. Todavia, a medição de desempenho, apesar de estar associada ao controle, não deve carregar um caráter opressor. $O$ momento atual exige, cada vez mais, formas efetivas de avaliar o desempenho da organização para servir como suporte às formas de aprendizado da organização e orientar a tomada de decisão, com o foco em sua estratégia (KYIAN, 2001).

\footnotetext{
${ }^{1}$ Esta pesquisa contou com o Auxílio Financeiro da Fundação de Amparo à Pesquisa do Rio Grande do Sul (FAPERGS).

Bacharel em Administração pela Universidade Federal do Rio Grande - FURG. Mestrando em Administração pelo Programa de Pós Graduação em Administração da Universidade Federal do Rio Grande (PPGA/FURG). E-mail: zepka@outlook.com

Doutora em Administração pela Universidade do Vale dos Sinos (UNISINOS). Professora Adjunta do Programa de Pós-Graduação em Administração da Universidade Federal do Rio Grande - PPGA/FURG. E-mail: liviacdavila@gmail.com.

"* Graduanda em Administração pela Universidade Federal do Rio Grande - FURG. E-mail: franciellepsilveira@yahoo.com.br
} 
As organizações definem sua estratégia, mas, como aponta Fischmann (1987), encontram dificuldades na fase de implementação. Precisam do controle, dos sistemas e dos indicadores de desempenho que tornem possíveis formas de decisão que permitam o gerenciamento adequado das competências internas da organização. Dessa forma, Fischmann (1999) conceitua desempenho como "a capacidade da empresa em atingir seus objetivos estratégicos através da implementação de estratégias adotadas dentro do seu processo de planejamento". Para o autor, as organizações necessitam de indicadores de desempenho que permitam a verificação do sucesso de sua gestão estratégica.

Para Hacker e Brotherton (1998), um sistema de indicadores efetivo precisa permitir e estimular a capacitação dos administradores da organização, a fim de determinar se as atividades programadas estão ocorrendo de fato e se elas estão atendendo os objetivos da organização. Yeo (2003) aponta para a necessidade de conectar os objetivos operacionais aos objetivos estratégicos.

Nesse contexto, é importante demonstrar que as organizações têm utilizado instrumentos que privilegiam o uso de indicadores financeiros, em detrimento de modelos que considerem outros aspectos não financeiros que também olhem aspectos internos da organização (BORTOLUZZI et al., 2011). Novas pesquisas têm surgido com o intuito de buscar e divulgar instrumentos que permitam indicar aspectos mais abrangentes do comportamento da organização, analisando-a por vários ângulos.

Pesquisa feira por Soares e de Melo (2008) procurou investigar e analisar, sob a ótica do desempenho organizacional, as publicações nacionais que discutissem formas de avaliar o desempenho das organizações através de indicadores de desempenho. Através da bibliometria, encontraram-se 26 artigos relevantes ao tema de pesquisa, publicados entre os anos 2000 a 2012, na base de dados Scielo. Em outro trabalho, Lacerda, Ensslin e Ensslin (2012) buscaram identificar como a avaliação de desempenho afetaria o desempenho da estratégia empresarial e poderia impactar o desempenho das organizações através de uma bibliometria nos artigos publicados entre 2000 e 2010. Identificou-se 41 artigos, coletados na base de dados Web of Science.

Dentro dessa perspectiva, um levantamento da produção científica sobre os indicadores de desempenho em serviços e produção torna-se relevante para o atual momento das organizações, a fim de proporcionar à área informações que possam demonstrar aos gestores das organizações as práticas identificadas para a gestão das informações relevantes na tomada de decisão.

O objetivo geral do presente artigo é caracterizar a produção científica sobre os indicadores de desempenho em serviços e produção, por meio de uma análise bibliométrica de artigos publicados no período entre os anos de 2007 e 2017. Para atingir o objetivo geral, este estudo teve, como direcionadores, os seguintes objetivos específicos:

(i) Levantamento da produção científica acerca do tema;

(ii) Análise bibliométrica dos artigos encontrados; e

(iii) Avaliação da produção científica sobre o tema através dos artigos encontrados.

O presente artigo encontra-se dividido em cinco seções. Logo após a introdução, a seção 2 apresenta a fundamentação teórica referente ao tema de pesquisa. Na sequência, a seção 3 demonstra a metodologia utilizada para a pesquisa, fundamentando a análise bibliométrica. A seção 4 detalha os procedimentos metodológicos da pesquisa. E, por último, a seção 5 apresenta os resultados e as considerações finais do presente artigo, bem como limitações encontradas e sugestões para futuras pesquisas.

\section{INDICADORES DE DESEMPENHO}

Há uma necessidade do alinhamento entre os sistemas produtivos e as prestações de serviços das empresas a seus objetivos estratégicos, para que as empresas permaneçam competitivas em um ambiente de extrema mudança, como é o ambiente de mercado atual. Todavia, conforme aponta Martins (1999), para que haja um melhoramento contínuo, é necessário que as empresas passem a acompanhar seus movimentos internos para perceber seus gargalos produtivos e aprimorá-los sempre.

Diante desse cenário, torna-se necessário que as organizações invistam em formas de medir o desempenho e construir indicadores de desempenho que possibilitem essa visão sistêmica da organização (BOND, 2002). A medição de desempenho é central na gestão das organizações, pois a mesma permite o direcionamento na tomada de decisão quanto à qualidade e a quantidades nos produtos e serviços prestados pelas organizações, conforme aponta Kaydos (1991). Nesse sentido, Neely et al., 1995 conceituam a medição de desempenho como sendo a técnica que se utiliza para quantificar a eficiência e a eficácia das atividades do negócio, em que a eficiência vai tratar da relação entre a utilização econômica dos recursos organizacionais, levando em consideração um determinado nível de satisfação. Já a eficácia, por sua vez, avalia o resultado de um processo cujas expectativas dos clientes são ou não atendidas.

Bond (2002) afirma que o desenvolvimento de planos de ações, novos programas ou mesmo mudanças nos sistemas produtivos são decididos baseados em indicadores que permitem, ao tomador de 
decisão, enxergar os estágios passados da organização, e, por consequência, permitir a criação de possíveis cenários futuros (Bond, 2002).

O objetivo de se construir indicadores de desempenho para a organização, reside na necessidade de ter um melhor conhecimento de seus processos, produtos e a forma como a prestação de serviços tem sido feita aos clientes (O'MARA et al., 1998). Ainda para os autores, a importância dos indicadores de desempenho vai além de controlar as atividades da organização, eles influenciam em todas as decisões tomadas dentro da mesma.

Desde o momento em que a missão da organização é definida e os objetivos planejados começam a ser colocados em prática, é importante verificar, rotineiramente, se a organização está alcançando aquilo que foi planejado (MÜLLER, 2003). Dessa forma, os indicadores de desempenho podem servir como ferramenta que auxilie no processo de alcançar os objetivos propostos, acompanhando os resultados e possibilitando uma visão clara do andamento dos processos na organização (ANTHONY e GOVINDARAJAN, 2008).

Os indicadores de desempenho precisam ser definidos baseados na estratégia da organização, avaliando o desempenho da mesma e possibilitando aos gestores das organizações que tomem decisões que se alinhem com as metas e objetivos propostos. (AGUIAR, 2012). Dessa forma, Frezatti et al. (2009) sustentam que os indicadores de desempenho precisam estar focados em identificar resultados que possam ser mensurados em uma organização durante um período pré-determinado de tempo, para que contribuam com a gestão eficaz da organização.

Todavia, para que se possa inferir através dos resultados obtidos nos indicadores de desempenho, é importante que os gestores tenham um claro entendimento de quais dados desejam coletar para que se possa medir, de formar adequada, e fornecer informações que serão efetivamente utilizadas na tomada de decisão (DICKINSON et al., 1997). Nesse direcionamento, Ferreira e Otley (2009) apontam que o gerenciamento do desempenho começa com a definição dos propósitos e objetivos. Dessa forma, Anthony e Govindarajan (2008) acrescentam que os indicadores de desempenho são um recurso que as organizações podem utilizar a fim de conseguir atingir os seus objetivos e implementar, de forma efetiva, a sua estratégia.

Bond (2002) aponta que as organizações estão reformulando seus sistemas de medição de desempenho, para que possam expandir suas análises para além de critérios financeiros, enxergando todo o contexto da organização, alinhando tudo a seus objetivos estratégicos. Os indicadores de desempenho são importantes ferramentas que auxiliam na medição da qualidade e desenvolvimento das atividades dos trabalhadores envolvidos nas tarefas das empresas (JACQUES e RASIA, 2016). Nesse sentido, Otley (2008) acrescenta que a medição de desempenho nas organizações tem sido uma tarefa com uma complexidade cada vez maior, há uma necessidade de atender a diversos objetivos simultaneamente e isso exige, das organizações, um olhar crítico para definirem quais os aspectos mais relevantes de sua organização que realmente precisam ser medidos.

Cardoza (2005) indica que os indicadores de desempenho precisam ser revistos sob essa nova ótica de mercado. Nesse sentido, Neely (1998) acrescenta que, no cenário empresarial atual, os novos modelos de avaliação de desempenho tornaram-se necessários para: verificar e comunicar a posição da empresa no mercado; motivar o progresso e o comprometimento dos funcionários através das mudanças e projetos de melhoria implantados; e para auxiliar na tomada de decisão gerencial sobre o processo de implantação e gerenciamento das melhorias e mudanças empresariais.

Bond (2002) finaliza, ao apontar que existe uma tendência de que as organizações, cada vez mais, construirão seus próprios indicadores e processos de medição de desempenho diferenciados, para permitir uma observação das peculiaridades da organização e, de forma detalhada, obter indicadores que demonstrem a realidade do que acontece na organização, em todos os seus níveis.

São vários os tipos de indicadores de desempenho utilizados pelas organizações a fim de obter as informações relevantes. Nas pesquisas realizadas, para construírem esse trabalho, encontraram-se como mais relevantes os modelos de: Balanced Scorecard (KAPLAN e NORTON, 1996), Integrated Performance Measurement Systems (BITITCI; CARRIE e MCDEVITT, 1997), Performance Prism (NEELY e ADAMS, 2001), e Performance Pyramid (CROSS e LYNCH, 1990). Foram escolhidos estes modelos com a finalidade caracterização, pois apresentam objetivos, áreas críticas de desempenho, processos e tipos de indicadores que precisam ser utilizados pelos administradores para medir o desempenho organizacional.

O contexto das organizações mudou durante os anos. Pech, Ramos, Favretto e Artifon (2015) mostram que, inicialmente, o foco das organizações era nos resultados, depois passou a ser na qualidade. Em tempos mais atuais, as organizações voltaram sua atenção à preocupação com mercados, clientes $e$ colaboradores para alcançar seus objetivos. É nesse contexto que Kaplan e Norton (1996) estudaram as formas de informar a gerência sobre as consequências de cada decisão tomada e como cada uma deveria estar focada na estratégia da organização, através de sua visão de futuro. Assim, os autores propõem o Balanced Scorecard (BSC), em que Silva (2013) define o BSC como uma ferramenta que permite descrever 
a estratégia da organização de forma clara, a partir de uma visão holística e balanceada, através de quatro perspectivas: financeira, de mercado, de processos internos e de aprendizagem e inovação. $\mathrm{O}$ autor conclui, ao afirmar que o BSC permite o alinhamento dos objetivos estratégicos com indicadores de desempenho, metas e planos de ação, integrando as quatro perspectivas, a gestão da organização pode voltar seus esforços à estratégia definida pela organização.

Irhamni, Khotimah e Rahmawati (2017) apresentam o modelo de medição de desempenho Integrated Performance Measurement System (IPMS), desenvolvido por Bititci, Carrie e McDevitt (1997). Os autores explicam que a estrutura desse modelo está baseada no desdobramento da visão organizacional até à medição de desempenho propriamente ditas. Nesse modelo, as medições de desempenho são feitas em vários níveis da organização, de forma que o desdobramento dos objetivos aconteça de forma consistente com a hierarquia e coerente com o impacto nas áreas de negócio individualmente. Assim, para que o IPMS funcione na empresa, é necessário que os processos executados pela empresa estejam de acordo com a estratégia e com os objetivos organizacionais. Dessa forma, o IPMS consegue integrar as várias áreas de negócio e desdobrar os objetivos do negócio através de toda a hierarquia da organização.

Maltempi (2014) conceitua o Performance Prism como um sistema de medição de desempenho em um modelo tridimensional, em formato de prisma de cinco fases. Nele, os stakeholders são colocados em primeiro plano e surgem cinco questões-chave, sendo elas: satisfação, estratégia, processos, capacidades e contribuições - prioridades que precisam ser trabalhadas para o atendimento das demandas dos stakeholders. Na satisfação, busca-se identificar quais as demandas dos stakeholders que precisam ser satisfeitas; na estratégica, busca-se entender quais as estratégias que serão definidas e implementadas para que as demandas dos stakeholders sejam atendidas. Quanto aos processos, definem-se quais os processos críticos que precisam ser executados para atender às estratégias traçadas. Com relação às capacidades, procura-se entender quais são as capacidades que precisam ser desenvolvidas ou melhoradas para intensificarem-se os processos críticos da empresa. Por fim, quanto às contribuições, identifica-se quais contribuições os stakeholders precisam fazer para que as capacidades sejam desenvolvidas ou melhoradas, conforme a necessidade da organização.

Hora e Viera (2008) identificam o Performance Pyramid como uma proposta de mudança da orientação das empresas dos custos para melhoria dos processos, levando a visão da organização através de seus objetivos financeiros e de mercado para todas as áreas da empresa. Para os autores, essa visão deverá mostrar onde a empresa está, aonde ela deseja chegar e quais meios serão necessários empregar para que se atinja esse objetivo. Os autores explicam que, dividindo os indicadores em dois grupos, sendo eles indicadores de eficácia externa (o próprio mercado) e indicadores de eficiência interna (os processos internos da empresa), a formalização da visão da organização permite um melhor gerenciamento da organização, a fim de garantir a obtenção dos resultados planejados.

Diante do cenário exposto, assuntos como indicadores de desempenho em serviços e produção podem ser estudados através de uma perspectiva do que se tem disponível nas bases de dados de produção científica.

\section{METODOLOGIA}

Os procedimentos metodológicos deste artigo apresentam-se baseados na estrutura proposta por Roesch (2013), descritos quanto ao seu propósito, caráter, delineamento e técnicas de coleta e técnicas de análise dos dados.

No que tange ao propósito, a pesquisa pode ser classificada como básica, por buscar um conhecimento como fim em si mesmo (ROESCH, 2013). Tal perspectiva se alinha com o objetivo geral deste estudo, pois visa caracterizar a produção científica brasileira sobre os indicadores de desempenho em serviços e produção, através de uma análise bibliométrica dos trabalhos publicados na última década.

Quanto ao caráter, a pesquisa pode ser classificada como quantitativa, pois converteu as informações dos artigos selecionados em dados estatísticos para análise. O delineamento adotado para esta pesquisa é a pesquisa descritiva, que, de acordo com Gil (2010), tem por objetivo a descrição das características de determinada população. Nesse estudo, a população consiste nos artigos que tratem da temática indicadores de desempenho em serviços e produção que foram selecionados para análise bibliométrica, através das bases de dados online.

A técnica de coleta de dados adotada foi a pesquisa bibliográfica, caracterizando-se como a fonte de dados secundários deste estudo. Segundo Gil (2010), a pesquisa bibliográfica é elaborada com base em materiais já publicados, tais como: livros, revistas, jornais, teses, dissertações e anais de eventos científicos.

Já a técnica de análise de dados foi a análise bibliométrica, para fundamentar o objetivo de pesquisa proposto e proporcionar o alcance do mesmo. Este método é composto de técnicas estatísticas e matemáticas, a fim de descrever aspectos da literatura e de outros meios de comunicação (ARAÚJO, 2006). Para Spinak (1996), bibliometria é o estudo da produção científica sobre o tema de pesquisa, através de 
aspectos quantitativos de produção, disseminação e uso da informação registrada com emprego de métodos matemáticos e estatísticos. Araújo e Alvarenga (2011) acrescentam que a pesquisa bibliométrica, através dos processos de levantamentos de dados, do tratamento destes e, depois, da apresentação dos dados ajuda os pesquisadores a entenderem o processo evolutivo do que é produzido em algum campo de estudos, de uma área específica. A bibliometria, para Sciasci et al. (2012), pode ser conceitualizada como "o estudo dos aspectos quantitativos da produção, disseminação, e uso da informação registrada, a partir de padrões e modelos matemáticos". Bastos e de Oliveira (2015) acrescentam que a bibliometria é a contagem de atividades bibliográficas, permite a possibilidade de estudo de termos específicos, como, por exemplo, autores, instituições, número de citações, entre outros pontos que possam ser de interesse do pesquisador.

Existem diversas teorias sobre a bibliometria. Wormell (1998) e Vanti (2002) destacam cinco principais tipos de metodologias utilizadas pela bibliometria: (i) análise de citações; (ii) análise de co-citação; (iii) agrupamento bibliográfico; (iv) co-word analysis; e (v) "Webometria". A teoria da bibliometria, abordada para fundamentar este estudo, está baseada em Longaray et al., (2015). Os autores fundamentam a pesquisa em três aspectos: (i) estudo da produção e autoria; (ii) estudo do conteúdo; e (iii) estudo das referências bibliográficas. O resultado da revisão sistemática da literatura sobre indicadores de desempenho em serviços e produção pode ser observado no quadro 1 a seguir.

QUADRO 1 - Portfólio Bibliométrico

\begin{tabular}{|c|c|}
\hline ID & Artigo \\
\hline 1 & $\begin{array}{l}\text { Abdullah, F., et al. (2011). "Bank Service Quality (BSQ) Index: An indicator of service } \\
\text { performance." International Journal of Quality and Reliability Management 28(5): } 542-555 .\end{array}$ \\
\hline 2 & $\begin{array}{l}\text { Blankson, C. and J. C. Crawford (2012). "Impact of positioning strategies on service firm } \\
\text { performance." Journal of Business Research } 65(3): 311-316 \text {. }\end{array}$ \\
\hline 3 & $\begin{array}{l}\text { Bunse, K., et al. (2011). "Integrating energy efficiency performance in production management } \\
- \text { Gap analysis between industrial needs and scientific literature." Journal of Cleaner } \\
\text { Production 19(6-7): } 667-679 \text {. }\end{array}$ \\
\hline 4 & $\begin{array}{l}\text { Choi, J., et al. (2017). "Data mining-based variable assessment methodology for evaluating the } \\
\text { contribution of knowledge services of a public research institute to business performance of } \\
\text { firms." Expert Systems with Applications } 84: 37-48 \text {. }\end{array}$ \\
\hline 5 & $\begin{array}{l}\text { Deflorio, F. P., et al. (2010). "Freight distribution performance indicators for service quality } \\
\text { planning in large transportation networks." Flexible Services and Manufacturing Journal } 22(1-2) \text { : } \\
36-60 \text {. }\end{array}$ \\
\hline 6 & $\begin{array}{l}\text { del Valle, I. D., et al. (2009). "The effects of training on performance in service companies A } \\
\text { data panel study." International Journal of Manpower } 30(3-4): 393-407 \text {. }\end{array}$ \\
\hline 7 & $\begin{array}{l}\text { Eisingerich, A. B., et al. (2014). "Acta Non Verba? The Role of Customer Participation and Word } \\
\text { of Mouth in the Relationship Between Service Firms' Customer Satisfaction and Sales } \\
\text { Performance." Journal of Service Research } 17(1): 40-53 \text {. }\end{array}$ \\
\hline 8 & $\begin{array}{l}\text { Franceschini, F., et al. (2008). "Properties of performance indicators in operations } \\
\text { management: A reference framework." International Journal of Productivity and Performance } \\
\text { Management } 57(2): 137-155 \text {. }\end{array}$ \\
\hline 9 & $\begin{array}{l}\text { Fu, N., et al. (2015). "How do high performance work systems influence organizational } \\
\text { innovation in professional service firms?" Employee Relations } 37(2): 209-231 .\end{array}$ \\
\hline 10 & $\begin{array}{l}\text { Gouscos, D., et al. (2007). "A general model of performance and quality for one-stop e- } \\
\text { Government service offerings." Government Information Quarterly } 24(4): 860-885 \text {. }\end{array}$ \\
\hline 11 & $\begin{array}{l}\text { Gupta, V., et al. (2013). "A strategic and operational approach to assess the lean performance } \\
\text { in radial tyre manufacturing in India: A case based study." International Journal of Productivity } \\
\text { and Performance Management } 62(6): 634-651 \text {. }\end{array}$ \\
\hline 12 & $\begin{array}{l}\text { Hassanein, A. A. G. and R. A. Khalifa (2007). "Financial and operational performance indicators } \\
\text { applied to public and private water and wastewater utilities." Engineering, Construction and } \\
\text { Architectural Management } 14(5): 479-492 \text {. }\end{array}$ \\
\hline 13 & $\begin{array}{l}\text { Hofmann, E. and K. Lampe (2013). "Financial statement analysis of logistics service providers: } \\
\text { ways of enhancing performance." International Journal of Physical Distribution \& Logistics } \\
\text { Management } 43(4): 321-342 \text {. }\end{array}$ \\
\hline 14 & $\begin{array}{l}\text { Kim, P. B. and K. D. Carlson (2016). "Agreement on service performance ratings between } \\
\text { frontline employees and their supervisor." Journal of Service Theory and Practice 26(5): } 721 \text { - } \\
740 \text {. }\end{array}$ \\
\hline 15 & $\begin{array}{l}\text { Kuo, Y.-F. and P.-C. Chen (2008). "Constructing performance appraisal indicators for mobility } \\
\text { of the service industries using Fuzzy Delphi Method." Expert Systems with Applications 35(4): } \\
1930-1939 .\end{array}$ \\
\hline
\end{tabular}




\begin{tabular}{|c|l|c|}
\hline 16 & $\begin{array}{l}\text { Laats, K., et al. (2011). "Performance measurement patterns in service companies An } \\
\text { empirical study on Estonian service companies." Baltic Journal of Management 6(3): 357-377. }\end{array}$ \\
\hline 17 & $\begin{array}{l}\text { Lin, Y.-T. and N.-C. Liu (2016). "High performance work systems and organizational service } \\
\text { performance: The roles of different organizational climates." International Journal of Hospitality } \\
\text { Management 55: 118-128. }\end{array}$ \\
\hline 18 & $\begin{array}{l}\text { Mansour, H., et al. (2013). "Evaluation of operational performance of workover rigs activities in } \\
\text { oilfields." International Journal of Productivity and Performance Management } 62(2): 204-218 .\end{array}$ \\
\hline 19 & $\begin{array}{l}\text { Menor, L. J. and A. V. Roth (2008). "New service development competence and performance: } \\
\text { An empirical investigation in retail banking." Production and Operations Management 17(3): 267- } \\
284 .\end{array}$ \\
\hline 20 & $\begin{array}{l}\text { Psomas, E. L., et al. (2013). "The impact of ISO 9001 effectiveness on the performance of } \\
\text { service companies." Managing Service Quality 23(2): 149-164. }\end{array}$ \\
\hline 21 & $\begin{array}{l}\text { Rocha, P. M. d., et al. (2016). "Analysis of the operational performance of brazilian airport } \\
\text { terminals: A multicriteria approach with De Borda-AHP integration." Journal of Air Transport } \\
\text { Management 51: 19-26. }\end{array}$ \\
\hline 22 & $\begin{array}{l}\text { Sawyerr, O. O., et al. (2009). "Call center employee personality factors and service } \\
\text { performance." Journal of Services Marketing 23(5): 301-317. }\end{array}$ \\
\hline 23 & $\begin{array}{l}\text { Sharma, M. G., et al. (2016). "Benchmarking of rail transport service performance through DEA } \\
\text { for Indian railways." International Journal of Logistics Management 27(3): 629-649. }\end{array}$ \\
\hline 24 & $\begin{array}{l}\text { Strezov, V., et al. (2013). "Defining sustainability indicators of iron and steel production." } \\
\text { Journal of Cleaner Production 51: } 66-70 .\end{array}$ \\
\hline 25 & $\begin{array}{l}\text { Tseng, M. L. (2013). "Modeling sustainable production indicators with linguistic preferences." } \\
\text { Journal of Cleaner Production 40: 46-56. }\end{array}$ \\
\hline 26 & $\begin{array}{l}\text { Tsikriktsis, N. (2007). "The effect of operational performance and focus on profitability: A } \\
\text { longitudinal study of the U.S. airline industry." Manufacturing and Service Operations } \\
\text { Management 9(4): 506-517. }\end{array}$ \\
\hline 27 & $\begin{array}{l}\text { Zhang, X., et al. (2017). "Effects of key enabling technologies for seru production on } \\
\text { sustainable performance." Omega 66, Part B: 290-307. }\end{array}$ \\
\hline
\end{tabular}

FONTE: Dados da pesquisa

\section{ANÁLISE BIBLIOMÉTRICA}

Esta seção consiste na apresentação do desenvolvimento das duas etapas da análise bibliométrica. Na primeira etapa, foi realizada a coleta dos dados referente ao propósito do estudo, delineado no objetivo geral da pesquisa. A segunda etapa propôs-se a apresentar os resultados atingidos a partir da análise dos dados coletados.

\subsection{Coleta de dados}

A coleta de dados iniciou na plataforma de buscas Google Scholar. Dos resultados obtidos, foi feita uma busca em periódicos disponibilizados pela Capes. As bases de dados escolhidas para a pesquisa foram escolhidas por sua relevância: Scopus, Web of Science e Science Direct.

Procurou-se fazer um levantamento da produção científica relacionada a indicadores de desempenho em serviços e produção. Foram feitas três pesquisas em cada base de dados: PERFORMANCE INDICATOR and OPERATIONAL; PERFORMANCE INDICATOR and PRODUCTION; P PERFORMANCE INDICATOR and SERVICE.

Na base de dados Google Scholar, foi procurado, no título, no resumo e nas palavras-chave (article, title, abstract, keyboard). As pesquisas resultaram em 2.040.000, 3.160.000 e 3.660 .000 artigos para as pesquisas de PERFORMANCE INDICATOR, com os conectivos "e" OPERATIONAL, PRODUCTION e SERVICE, respectivamente.

Após observarem-se as quantidades de artigos resultantes no Google Scholar, as bases de dados escolhidas para a coleta de artigos foram Scopus, Web of Science e Science Direct. Justifica-se a escolha por essas três em Souza e Ribeiro (2009), cujos autores apontam que estas bases contemplam um número relevante de periódicos e englobam um número significativo de pesquisas que se relacionam com o assunto de interesse devidamente delimitado.

$\mathrm{Na}$ base de dados Scopus, procurou-se no título, no resumo e nas palavras-chave (article, title, abstract, keyboard), dentro da área de Negócios, Administração e Contabilidade (Business, Management and Accounting). Resultando-se em um total de 23.521 artigos. Já, na base de dados Web of Science, procurou-se nos tópicos (topic); dentro da área de ciências sociais (social sciences citation index), obteve-se 
519 artigos. Por fim, na base de dados Science Direct, procurou-se nos resumos, nos títulos e nas palavraschave (abstract, title, keyword); dentro da área de Negócios, Administração e Contabilidade (Business, Management and Accounting), resultando em 178 artigos. Pode-se observar um detalhamento maior na Figura 1 a seguir.

FIGURA 1 - Pesquisas feitas nas bases de dados

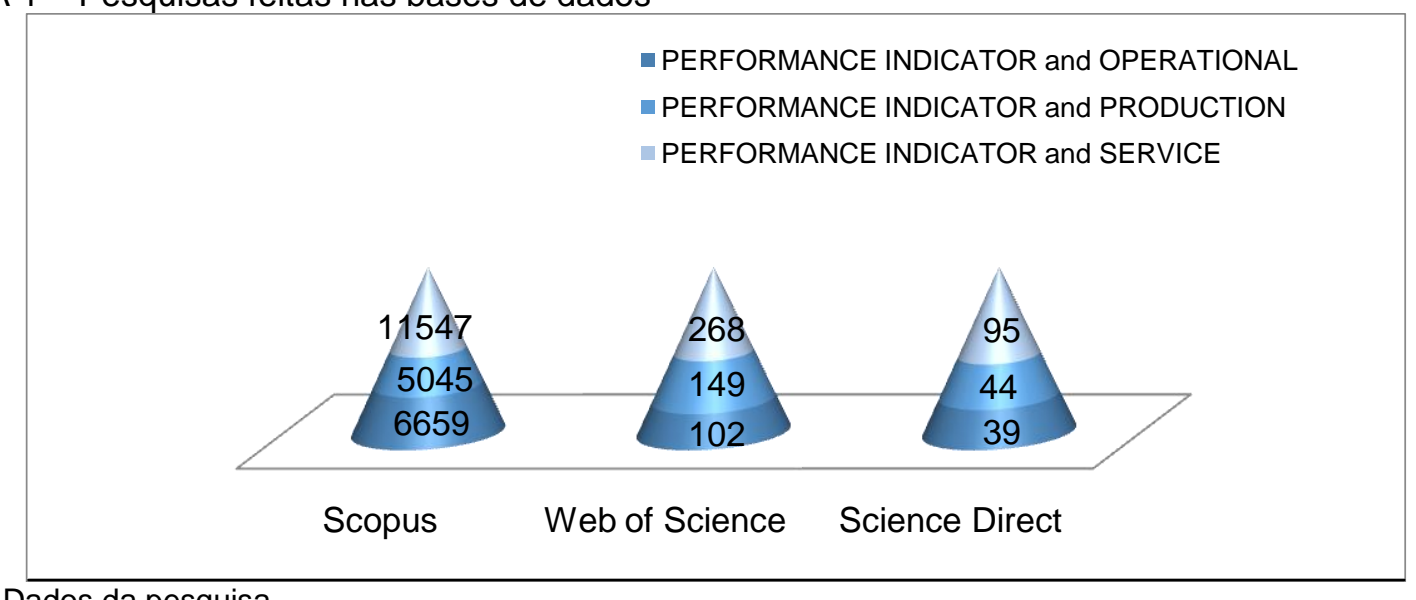

FONTE: Dados da pesquisa

A primeira análise de dados feita, depois de mesclarem-se os resultados obtidos nas bases de dados, foi os artigos repetidos e os não dentro do período de 2007 e 2017. Desses, ficaram 2104, 2156 e 2308 artigos para operacional, production e service. $\mathrm{Na}$ análise seguinte, foram retirados os artigos que não possuíam ligação com a proposta da pesquisa, através da análise individual de cada abstract. A primeira etapa, a de coletas de dados, finalizou com uma população total de 27 artigos.

TABELA 1 - Análise dos artigos de acordo com a proposta de pesquisa

\begin{tabular}{c|l|c}
\hline Pesquisa & Termos procurados & Resultados \\
\hline 1 & PERFORMANCE INDICATOR and OPERATIONAL & 9 artigos \\
\hline 2 & PERFORMANCE INDICATOR and PRODUCTION & 7 artigos \\
\hline 3 & PERFORMANCE INDICATOR and SERVICE & 11 artigos
\end{tabular}

FONTE: dados da pesquisa

\section{RESULTADOS}

A fim de analisar os resultados obtidos na primeira fase, o resultado foi divido em categorias: o estudo acerca da produção e autoria, o estudo acerca do conteúdo e o estudo acerca das referências bibliográficas.

\subsection{Estudo da produção e autoria}

Ao estudar-se produção e autoria, o objetivo foi levantar o número de publicações por ano e considerar os artigos através do número de autores dos artigos. Além disso, buscou-se identificar os autores mais produtivos no assunto. Para que se mensurasse o progresso da produção científica da produção analisada com relação a indicadores de performance em serviços e produção, construiu-se um histograma de acordo com o número de publicações identificadas a cada ano, assim como sua respectiva linha de tendência linear, exposta na figura 2. Assim, foi adotada uma curva de melhor ajuste usando como critério o maior valor de $\mathrm{R}^{2}$. Nesse escopo, o valor de $\mathrm{R}^{2}$ aponta o percentual de variação da variável dependente (número de artigos), explicada pela variável independente (ano). 
FIGURA 2 - Publicações por ano

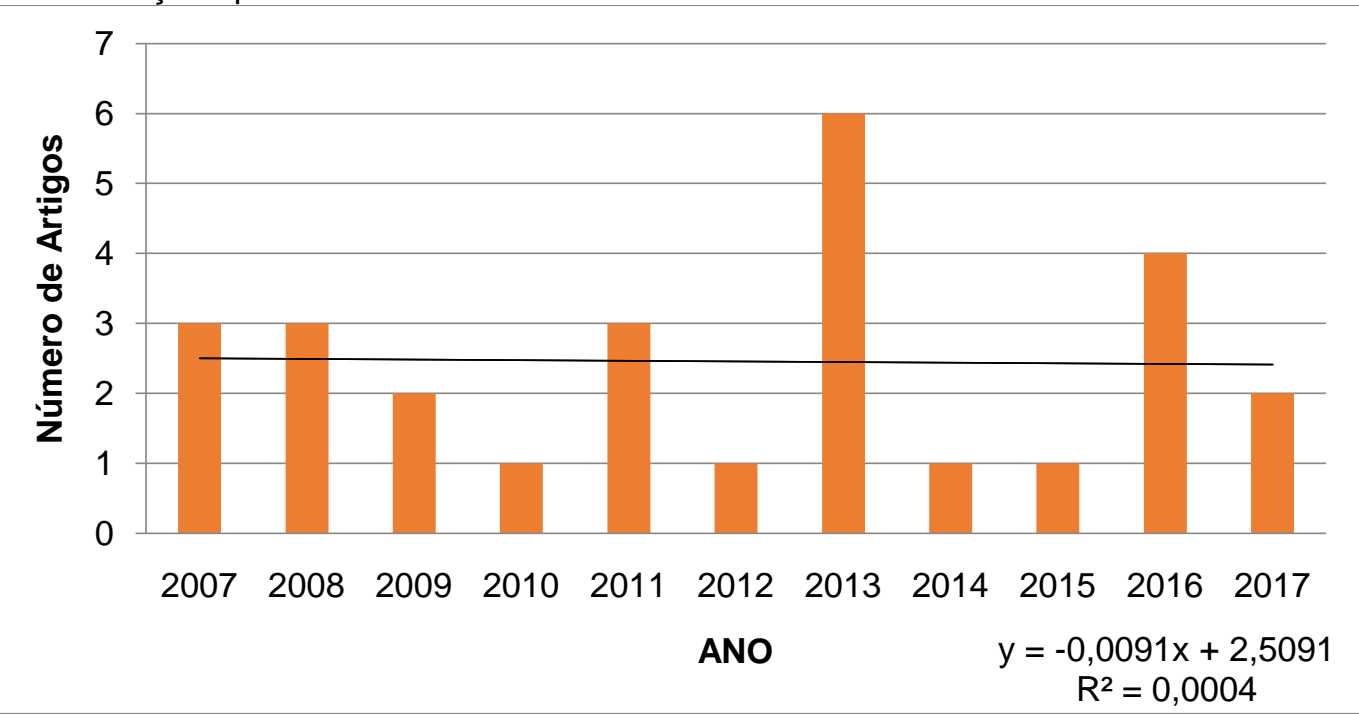

FONTE: dados da pesquisa

Constata-se que a produção da amostra, no período analisado, apresenta um coeficiente de determinação de 0.0004. Percebeu-se uma relativa constância de publicações acerca dos temas pesquisados durante os anos de 2007 e 2017, destacando-se o ano de 2013 com 6 publicações na composição da amostra.

$\mathrm{Na}$ sequência, classificou-se a população de artigos com relação ao número de autores por artigo, verificando-se que artigos, com apenas um autor, representam 7,4\%; a maioria dos artigos são realizados por três ou quatro autores $(77,77 \%)$, e a menor parte dos artigos analisados são realizados entre cinco ou sete autores $(14,81 \%)$. É interessante ressaltar que não há artigos com autoria de seis autores. Os dados podem ser visualizados na tabela 2 .

TABELA 2 - Número de autores por artigo

\begin{tabular}{c|c|c}
\hline Número de autores & Artigos & Percentual de artigos \\
\hline 1 autor & 2 artigos & $7,4 \%$ \\
\hline 2 autores & 7 artigos & $25,92 \%$ \\
\hline 3 autores & 8 artigos & $29,62 \%$ \\
\hline 4 autores & 6 artigos & $22,22 \%$ \\
\hline 5 autores & 3 artigos & $11,11 \%$ \\
\hline 6 autores & 0 artigos & $0 \%$ \\
\hline 7 autores & 1 artigos & $3,7 \%$ \\
\hline
\end{tabular}

FONTE: dados da pesquisa

A fim de contabilizar os autores produtivos no assunto estudado, elencaram-se os autores com mais participações na amostra de artigos, optou-se pela contagem absoluta de autoria, isto é, no caso de um artigo produzido por dois ou mais pesquisadores, atribuiu-se uma frequência para cada um. Foram encontrados um total de 68 autores para os 27 artigos, com uma média de 2,5 autores por artigo. As especificações dessa parte da pesquisa podem ser observadas na tabela 7. Todavia, não foi possível concluir-se quais os autores mais produtivos, nenhum dos autores estudados produziu mais do que um artigo na composição da amostra.

Ao finalizar o estudo sobre a autoria, foram encontradas as instituições com maior produtividade científica acerca do tema. Foi atribuída 1 frequência por instituição, ainda que este autor estivesse vinculado a mais de uma, chegando-se ao total de 83 frequências. Destas, ao analisar-se, foram destacadas as 10 mais produtivas, com frequência de produção maior do que 2 publicações. As instituições mais produtivas foram Korea Institute of Science and Technology Information (da Coréia) e Politecnico di Torino (da Itália), ambos com 5 publicações cada, somando $12 \%$ de toda a publicação. Na tabela 3, apresentam-se as 10 instituições mais produtivas, que, juntas, somam $43 \%$ de toda a publicação da população do portfólio bibliométrico. 
TABELA 3 - Instituições mais produtivas

\begin{tabular}{c|c|c|c}
\hline $\begin{array}{c}\text { Instituição } \\
\text { Korea Institute of Science and Technology } \\
\text { Information }\end{array}$ & País & $\begin{array}{c}\text { Número de } \\
\text { recorrências }\end{array}$ & $\begin{array}{c}\text { Percentual de } \\
\text { artigos }\end{array}$ \\
\hline Politecnico di Torino & 5 vezes & $6 \%$ \\
\hline Teesside University & Itália & 5 vezes & $6 \%$ \\
\hline Universidade Federal Fluminense & Reino Unido & 4 vezes & $4,8 \%$ \\
\hline BWI Center for Industrial Management & Brasil & 4 vezes & $4,8 \%$ \\
\hline Salifornia State Polytechnic University & Estados Unidos & 3 vezes & $3,6 \%$ \\
\hline Macquarie University & Austrália & 3 vezes & $3,6 \%$ \\
\hline Universidad Complutense de Madrid & Itália & 3 vezes & $3,6 \%$ \\
\hline Universiti Teknologi Mara & Malásia & 3 vezes & $3,6 \%$ \\
\hline University of St Gallen & Suíça & 3 vezes & $3,6 \%$ \\
\hline
\end{tabular}

FONTE: dados da pesquisa

\subsection{Estudo do conteúdo}

Através da análise de conteúdo dos artigos, procurou-se identificar quais seriam os temas mais recorrentes publicados no período de 2007 a 2017. Foi feita a análise dos temas de pesquisa e classificaram-se quais seriam os temas mais abordados nas pesquisas. Logo após, foi feita a análise das palavras-chave que apareceram em uma quantidade maior nos artigos.

Percebeu-se que o tema mais frequente foi os estudos sobre indicadores de desempenho em empresas prestadoras de serviços, com um total de 9 artigos (33,3\%), seguido de estudos sobre indicadores de desempenho em processos produtivos, com 8 artigos $(29,2 \%)$. Indicadores de qualidade em serviços e desempenho financeiro em processos produtivos aparecem no fim, com 7 (25\%) e 3 artigos (12,5\%), respectivamente. Esta análise pode ser percebida na tabela 4.

TABELA 4 - Resumo dos temas abordados nos artigos selecionados

\begin{tabular}{l|c|c}
\multicolumn{1}{c|}{ Temas } & Número de artigos & Percentual de artigos \\
\hline $\begin{array}{l}\text { Indicadores de desempenho em empresas prestadoras } \\
\text { de serviços }\end{array}$ & 9 artigos & $33.30 \%$ \\
\hline Indicadores de desempenho em processos produtivos & 8 artigos & $29.20 \%$ \\
\hline Indicadores de qualidade em serviços & 7 artigos & $25 \%$ \\
\hline Desempenho financeiro em processos produtivos & 3 artigos & $12.50 \%$ \\
\hline
\end{tabular}

FONTE: dados da pesquisa

Feita a análise dos temas através dos resumos dos artigos, desenvolveu-se a análise das palavraschave mais utilizadas nos artigos, em que foi contabilizado um total de 134 palavras-chave. Nesse sentido, as palavras-chave que apareceram mostraram um relação direta com as palavras pesquisadas nas bases de dados no momento inicial. Assim, as palavras em que se teve uma recorrência maior foram "Performance measurement" e "Sustainable production indicators", com um total de 4 recorrências cada, representando 2,98\% cada uma, do total de palavras-chave. Logo depois, aparecem "Financial statements", "Quality indicators" e "Service industries, representando 3 recorrências cada uma, com uma porcentagem de $2,23 \%$ cada. Os dados podem ser vistos na tabela 5 .

TABELA 5 - Resumo das principais palavras-chave identificadas nos artigos

\begin{tabular}{c|c|c}
\hline Palavras-chave & $\begin{array}{c}\text { Número de } \\
\text { recorrências }\end{array}$ & Percentual \\
\hline Performance measurement & 4 & $2,98 \%$ \\
\hline Sustainable production indicators & 4 & $2,98 \%$ \\
\hline Financial statements & 3 & $2,23 \%$ \\
\hline Quality indicators & 3 & $2,23 \%$ \\
\hline Service industries & 3 & $2,23 \%$ \\
\hline
\end{tabular}

FONTE: dados da pesquisa 


\subsection{Estudo das referências bibliográficas}

Ao analisar as referências bibliográficas dos 27 artigos do portfólio bibliométrico, teve-se como objetivo identificar as obras de maior repercussão. Para isso, foi realizada a análise de todas as 1.417 referências para selecionar as que são relacionadas a pessoas físicas. Tomou-se como ponto de corte mais do que 3 citações por obra. Assim, a tabela 6 apresenta as obras de maior repercussão, destacando os autores, o nome da obra, o ano em que foi publicada, o local da publicação, o tipo da mesma e a quantidade de citações entre os artigos do portfólio.

TABELA 6 - Obras de maior repercussão entre os artigos selecionados

\begin{tabular}{|c|c|c|c|c|c|}
\hline Autores & Obra & Ano & Referência & Tipo & Citações \\
\hline BAGOZZI, R.; YI, Y. & $\begin{array}{c}\text { On the evaluation of } \\
\text { structural equation } \\
\text { models. }\end{array}$ & 1988 & $\begin{array}{c}\text { Journal of the } \\
\text { Academy of } \\
\text { Marketing Science } \\
\text { March 1988, Volume } \\
\text { 16, Issue 1, pp 74-94 }\end{array}$ & Artigo & 4 \\
\hline $\begin{array}{l}\text { PODSAKOFF, P. M.; } \\
\text { MACKENZIE; S. B.; } \\
\text { PODSAKOFF, N. P. }\end{array}$ & $\begin{array}{l}\text { Common method } \\
\text { biases in behavioral } \\
\text { research: A critical } \\
\text { review of the } \\
\text { literature and } \\
\text { recommended } \\
\text { remedies }\end{array}$ & 2003 & $\begin{array}{c}\text { Journal of Applied } \\
\text { Psychology 2003, } \\
\text { Vol. 88, No. 5, 879- } \\
903\end{array}$ & Artigo & 3 \\
\hline $\begin{array}{l}\text { ARMSTRONG, J. S; } \\
\text { OVERTON, T. S. }\end{array}$ & $\begin{array}{l}\text { Estimating } \\
\text { nonresponse bias in } \\
\text { mail surveys }\end{array}$ & 1977 & $\begin{array}{c}\text { Journal of Marketing } \\
\text { Research, Volume } \\
\text { 14, Issue 3, August } \\
\text { 1977, pages 396-402 }\end{array}$ & Artigo & 3 \\
\hline $\begin{array}{l}\text { PODSAKOFF, P. M; } \\
\text { ORGAN, D. W. }\end{array}$ & $\begin{array}{l}\text { Self-reports in } \\
\text { organizational } \\
\text { research: problems } \\
\text { and prospects }\end{array}$ & 1986 & $\begin{array}{c}\text { Journal of } \\
\text { Management, } \\
\text { December } 1986\end{array}$ & Artigo & 3 \\
\hline
\end{tabular}

FONTE: dados da pesquisa

Para finalizar este estudo bibliométrico, buscou-se identificar quais, dentre todos os artigos analisados no portfólio bibliométrico, são de maior relevância. Para isso, foi feito o uso do modelo utilizado por Longaray et al., (2015). Este modelo consiste na observação de dois indicadores, sendo eles o número de citações do artigo no Google Scholar e o número de citações nas próprias referências bibliográficas dos artigos do portfólio bibliométrico. Quando é feita a busca por um artigo no site Google Scholar, este mostra o número de vezes em que esse foi citado em outros artigos. Utilizando-se dessa ferramenta, a fim de constituir este primeiro indicador, foram pesquisados no site cada um dos 27 artigos, no dia 28 de outubro de 2017. No que se refere ao segundo indicador, foi obtido por meio da busca e contagem das citações nas próprias referências dos artigos selecionados no portfólio bibliométrico.

Indica-se, na tabela 7, a lista dos 9 artigos de maior relevância. A escolha foi feita baseando-se nos artigos que tivessem, no mínimo, 50 citações ao total. Apresenta-se, na primeira coluna, o título e a fonte do artigo; na segunda, o número de citações no Google Scholar "GS"; na terceira, o número de citações nas referências bibliográficas "RB" e, por fim, na quarta, o somatório " $\Sigma$ " do número de citações.

TABELA 7 - Artigos selecionados em ordem decrescente de relevância

\begin{tabular}{|c|c|c|c|}
\hline Artigo & GS & RB & $\Sigma$ \\
\hline $\begin{array}{l}\text { Bunse, K., et al. (2011). "Integrating energy efficiency performance in production } \\
\text { management - Gap analysis between industrial needs and scientific literature." } \\
\text { Journal of Cleaner Production } 19(6-7): 667-679 .\end{array}$ & 416 & 0 & 416 \\
\hline $\begin{array}{l}\text { Kuo, Y.-F. and P.-C. Chen (2008). "Constructing performance appraisal indicators } \\
\text { for mobility of the service industries using Fuzzy Delphi Method." Expert Systems } \\
\text { with Applications 35(4): 1930-1939. }\end{array}$ & 174 & 0 & 174 \\
\hline $\begin{array}{l}\text { Menor, L. J. and A. V. Roth (2008). "New service development competence and } \\
\text { performance: An empirical investigation in retail banking." Production and } \\
\text { Operations Management } 17(3): 267-284 \text {. }\end{array}$ & 170 & 0 & 170 \\
\hline
\end{tabular}




\begin{tabular}{|c|c|c|c|}
\hline $\begin{array}{l}\text { Gouscos, D., et al. (2007). "A general model of performance and quality for one- } \\
\text { stop e-Government service offerings." Government Information Quarterly } 24(4) \text { : } \\
860-885 \text {. }\end{array}$ & 145 & 0 & 145 \\
\hline $\begin{array}{l}\text { Abdullah, F., et al. (2011). "Bank Service Quality (BSQ) Index: An indicator of } \\
\text { service performance." International Journal of Quality and Reliability Management } \\
28(5): 542-555 \text {. }\end{array}$ & 97 & 1 & 98 \\
\hline $\begin{array}{l}\text { Tseng, M. L. (2013). "Modeling sustainable production indicators with linguistic } \\
\text { preferences." Journal of Cleaner Production 40: 46-56. }\end{array}$ & 84 & 0 & 84 \\
\hline $\begin{array}{l}\text { Psomas, E. L., et al. (2013). "The impact of ISO } 9001 \text { effectiveness on the } \\
\text { performance of service companies." Managing Service Quality 23(2): 149-164. }\end{array}$ & 78 & 0 & 78 \\
\hline $\begin{array}{l}\text { Sawyerr, O. O., et al. (2009). "Call center employee personality factors and service } \\
\text { performance." Journal of Services Marketing 23(5): } 301-317 \text {. }\end{array}$ & 74 & 1 & 75 \\
\hline $\begin{array}{l}\text { Tsikriktsis, N. (2007). "The effect of operational performance and focus on } \\
\text { profitability: A longitudinal study of the U.S. airline industry." Manufacturing and } \\
\text { Service Operations Management 9(4): } 506-517 \text {. }\end{array}$ & 74 & 1 & 75 \\
\hline
\end{tabular}

FONTE: dados da pesquisa

Legenda:

GS: Número de citações no Google Scholar.

RB: Número de citações entre as referências bibliográficas dos artigos do portfólio bibliométrico.

$\Sigma$ : somatório entre as citações no Google Scholar e as citações nas referências bibliográficas do portfólio bibliométrico.

Verificou-se que, dos 27 artigos selecionados, apenas 9 artigos obtêm um número maior do que 50 citações cada. Os 18 artigos seguintes representam 18,5\% das citações. Aos 33,3\% (9 artigos), atinge-se $81,5 \%$ das citações.

\section{CONCLUSÃO}

O estudo, atentando ao objetivo formulado, analisou a caracterização da produção científica relacionada aos indicadores de desempenho em serviços e produção, em artigos publicados no período entre 2007 e 2017, coletados nas bases de dados Science Direct, Scopus e Web of Science. Para isso, utilizaram-se as técnicas de análise bibliométrica, baseadas nos autores Spinak (1996), Wormell (1998), Vanti (2002), Araújo e Alvarenga (2011), Sciaci et al. (2012), Oliveira (2015), Longaray et al. (2015), e Oliveira (2015).

Foram analisados 27 artigos com maior relevância no assunto acerca de suas produções, autorias, conteúdos e referências. Entre os principais resultados encontrados na análise, foi possível constatar que os temas mais pesquisados nos artigos foram a busca por indicadores de desempenho em empresas prestadoras de serviços e processos produtivos. De maneira geral, observa-se que essas informações são similares a informações divulgadas em outros estudos evidenciados na fundamentação teórica, cujos indicadores de desempenho em serviços podem ser vistos em Bond (2002) e Bortoluzzi et al. (2001), e indicadores de desempenho em processos produtivos podem ser vistos em Müller (2003) e Cardoza e Carpinetti (2015).

A maioria dos artigos analisados tiveram por autoria entre 2 e 4 autores. Percebeu-se, também, que as instituições mais produtivas sobre indicadores de desempenho em empresas prestadoras de serviço e processos produtivos foram Korea Institute of Science and Technology Information, da Coréia, e o Instituto Politecnico di Torino, da Itália.

Os resultados deste trabalho podem facilitar o entendimento de que esses tipos de pesquisa agregam valor, sobretudo no tocante à disseminação de dados e informações que ajudam a compreender como as empresas de produção ou prestadoras de serviço estão atuando para medir e avaliar seus indicadores de desempenho. Além de difundir as informações que influenciam, de maneira macro, no crescimento do citado campo na literatura acadêmica internacional.

Dentre as limitações da pesquisa, destaca-se não terem sido encontrados artigos brasileiros, tendo em vista que as bases de dados trabalhadas são internacionais. Feita a revisão sistemática da literatura por meio da bibliometria, identificou-se, como lacuna de pesquisa, o número limitado de estudos que versem sobre indicadores de desempenho em serviços e produção, e a possibilidade de executar a mesma pesquisa em bases de dados brasileiras, a fim de caracterizar a produção nacional acerca do tema.

Como sugestão para futuras pesquisas, podem ser desenvolvidas, ainda na mesma temática, estender-se a um número maior de bases de dados e procurar outras palavras-chave também relacionadas ao tema. Outra possibilidade é o estudo dos artigos que estão como referências bibliográficas nos artigos do portfólio bibliométrico, demonstrando aqueles de maior relevância. 


\section{REFERÊNCIAS}

AGUIAR, A. B.; TEIXEIRA, A. J. C.; NOSSA, V.; GONZAGA, R. P. Associação entre sistema de incentivos gerenciais e práticas de contabilidade gerencial. RAE - Revista de Administração de Empresas, 52(1), 40-54. 2012. ANTHONY, R. N.; GOVINDARAJAN, V. Sistemas de controle gerencial. 12 ed. São Paulo: McGraw- Hill. 2008 ARAÚJO, C. A. Bibliometria: evolução histórica e questões atuais. Em Questão, v. 12, n. 1, p. 11- $32,2006$. ARAÚJO, R. F.; ALVARENGA, L. A bibliometria na pesquisa científica da pós-graduação brasileira de 1987 a 2007. Encontros Bibli: revista eletrônica de biblioteconomia e ciência da informação, v. 16, n. 31, 2011.

BASTOS, M. H. R.; de OLIVEIRA, U. R. Análise de discurso e Análise de Conteúdo: Um breve levantamento bibliométrico de suas aplicações nas ciências sociais aplicadas da Administração. In XII Simpósio de excelência em gestão e tecnologia. 28, 29 e 30 de Outubro de 2015 - Resende - Rio de Janeiro.

BITITCI, U.S., CARRIE, A.S.; MCDEVITT, L. Integrated performance measurement systems: a development guide. Int. J. Op. \& Prod. Management, v.17, n.5, p.522-534, 1997.

BOND, E. Medição de desempenho para gestão da produção em um cenário da cadeia de suprimentos. Dissertação (Mestrado) - Escola de Engenharia de São Carlos, Universidade de São Paulo, São Carlos, 2002.

BORTOLUZZI, S. C.; ENSSLIN, S. R.; ENSSLIN, L. Avaliação de desempenho multicritério como apoio à gestão de empresas: aplicação em uma empresa de serviços. Gest. Prod., São Carlos, v. 18, n. 3, p. 633-650, 2011.

CARDOZA, E.; CARPINETTI, L. C. R. Indicadores de desempenho para o sistema de produção enxuto. Revista Produção Online. ISSN 1676 - 1901. Vol. 5. Num. 2/ Junho de 2005.

CROSS, K. F.; LYNCH, R .L. Managing the corporate warriors. Quality progress, v.23, n.4, p.54-59, 1990.

DICKINSON T. L.; MCINTYRE R.M. A conceptual framework for teamwork measurement. In: Team performance assessment and measurement. Mahwah, NJ: Erlbaum, 1997.

FERREIRA, A.; OTLEY, D. The design and use of performance management systems: An extended framework for analysis. Management Accounting Research, 20(4), 263-282. doi:10.1016/j.mar.2009.07.003.

FISCHMANN, A. A. Implementação de estratégias: identificação e análise de problemas. 1987, Tese (Doutorado) Faculdade de Economia, Administração e Contabilidade, Universidade de São Paulo, São Paulo, 1987.

FISCHMANN, A. A.; ZILMER, A. M. Utilização de indicadores de desempenho como instrumento de suporte à gestão estratégica. In: XXIII Encontro da Associação Nacional de Pós-Graduação em Administração (ENANPAP), 1999.

FREZATTI, F.; ROCHA, W.; NASCIMENTO, A. R.; JUNQUEIRA, E. Controle Gerencial: uma abordagem da contabilidade gerencial no contexto econômico, comportamental e sociológico. São Paulo: Atlas. 2009.

GIL, A. C. Como elaborar projetos de pesquisa. 5.ed. São Paulo: Atlas, 2010.

HACKER, M. E., BROTHERSON, P. A. Designing and installing effective performance measurement systems. IIE Solutions, Vol. 30, № 8, pp18-23, Aug. 1998.

HORA, H. R. M.; VIERA, L. E. V. Sistemas de medição de desempenho organizacional: evolução e atualidade. Perspectivas online. Volume 5, número 1, 2008.

IRHAMNI, F. KHOTIMAH, B. K.; RAHMAWATI, D. Improvement Integrated Performance Measurement System (IPMS) for small and medium enterprise impact of information technology. Journal of Theoretical and Applied Information Technology 31st January 2017. Vol.95. No.2

JACQUES, F. V. S.; RASIA, K. A. Avaliação de desempenho organizacional: uma reflexão sobre o desempenho dos gestores. SINERGIA, Rio Grande, 20 (2): 59-72, 2016

KAYDOS, W. Measuring, managing and maximizing performance. Portland, Productivity Press. 1991

KAPLAN, R.S.; NORTON, D.P. Using the balanced scorecard as a strategic management system. Harvard business review, v.74, n.1, p.75-85, Jan./Feb, 1996.

KIYAN, F. M. Proposta para desenvolvimento de indicadores de desempenho como suporte estratégico. Dissertação (Mestrado) - Escola de Engenharia de São Carlos. Universidade de São Paulo, São Carlos, 2001.

LACERDA, R. T. D. O., ENSSLIN, L., \& ENSSLIN, S. R. (2012). Uma análise bibliométrica da literatura sobre estratégia e avaliação de desempenho. Gestão \& Produção, 19(1).

LONGARAY, A. A.; POPIOLEK JÚNIOR, T. L.; MUNHOZ, P. R.; GERI, F. S.; CASTELLI, T. M. Caracterização da produção científica brasileira sobre a aplicação de métodos multicritério de apoio à decisão: uma análise das publicações entre 2004 - 2013. In: XXXV Encontro Nacional de Engenharia de Produção (ENEGEP) , 2015.

MALTEMPI, M. M. Sistemas de medição de desempenho organizacional: uma análise comparativa das ferramentas Prism, Pyramid e BSC. Universitas - Ano 7 - № 13 - Julho/Dezembro 2014

MARTINS, R. A. Sistemas de medição de desempenho: Um modelo para estruturação do uso. Tese (Doutorado) Escola Politécnica. Universidade de São Paulo, São Paulo, 1999.

MÜLLER, C. J. Modelo de gestão integrando planejamento estratégico, sistemas de avaliação de desempenho e 
gerenciamento de processos (MEIO - Modelo de Estratégia, Indicadores e Operações). Tese (Doutorado) - Escola de Engenharia. Universidade Federal do Rio Grande do Sul, Porto Alegre, 2003

NEELY, A., GREGORY, M.; PLATTS, K. Performance measurement system design: a literature review and research agenda. International Journal of Operations \& Production Management, Vol. 15 No. 4, pp. 80-116. Mar, 1995.

NEELY, A. Measuring business performance. The Economics, London, c.3, 1998.

NEELY, A.; ADAMS, C. Perspectives on performance: The performance Prism. Center for business performance, Cranfield, UK, 2001.

O'MARA, C. E.; HYLAND, P. W.; CHAPMAN, R. L. Performance measurement and strategic change. Managing Service Quality: An International Journal, Vol. 8 Issue: 3, pp.178-182. Set. 1998.

OTLEY, D. Did Kaplan and Johnson get it right? Accounting, Auditing \& Accountability Journal2, 21(2), $229-239$. 2008.

PECH, L. M.; RAMOS, F. M.; FAVRETTO, J.; ARTIFON, R. L. Utilização do Balanced Scorecard em ambientes cooperativistas: um estudo de caso. REUNA, Belo Horizonte - MG, Brasil, v.20, n.3, p. 05-26, Jul. - Set. 2015 - ISSN 2179-8834

$\mathrm{ROESCH}$, S. M. A. Projetos de estágio e de pesquisa em administração: guia para estágios, trabalhos de conclusão, dissertações e estudos de caso. $3^{\underline{a}}$ ed. $-6^{\underline{a}}$ reimp. - São Paulo: Atlas, 2010.

SCIASCI, V.; GARCIA, S. F. A.; GALLI, L. C. D. L. A. Posicionamento de Marcas Globais: Um Estudo Bibliométrico da Produção Científica na Área. Revista Brasileira de Marketing, v. 11, n. 2, p. 67-93, 2012.

SILVA, D. D. O. Monitoramento e controle da estratégia baseado na ferramenta do Balanced Scorecard: Estudo de caso. Monografia (Especialização) Centro de Ensino Superior do Ceará. Faculdade Cearense. Fortaleza, 2013.

SOARES, T. C.; DE MELO, P. A. Avaliação de desempenho organizacional: um mapeamento em periódicos nacionais. Revista Eletrônica de Estratégia \& Negócios, v. 7, n. 2, p. 148-164, 2014.

SPINAK, E. Diccionario enciclopédico de bibliometría, cienciometría e informática. Unesco, 1996.

SOUZA, M. R.; RIBEIRO, A. L. P. (2009). Systematic review and meta-analysis of diagnostic and prognostic studies: a tutorial. Arquivo Brasileiro de Cardiologia, 92(3), 241-251.

WORMELL, I. Informetria: explorando bases de dados como instrumentos de análise. Ciência da Informação, Brasília, v.27, n. 2, p. 210-216, maio/ago. 1998.

VANTI, N. Da bibliometria à webometria: uma exploração conceitual dos mecanismos utilizados para medir o registro da Informação e a difusão do conhecimento. Ciência da Informação., Brasília, v. 31, n. 2, p. 152-162, maio/ago. 2002.

YEO, R. The tangibles and intangibles of organisational performance. Team Performance Management, v. 9, n. 7-8, p. 199-204, 2003. 
